\title{
Travelers as a Sentinel Population: Use of Sentinel Networks to Inform Pretravel and Posttravel Evaluation
}

\author{
Karin Leder, MBBS, FRACP, PhD, MPH, DTMH
}

Corresponding author

Karin Leder, MBBS, FRACP, PhD, MPH, DTMH

Victorian Infectious Disease Service, Royal Melbourne Hospital, Royal Parade, Parkville, Victoria, 3052, and Department of Epidemiology and Preventive Medicine, Monash University, Melbourne, 3004, Australia.

E-mail: karin.leder@med.monash.edu.au

Current Infectious Disease Reports 2009, 11:51-58

Current Medicine Group LLC ISSN 1523-3847

Copyright (C) 2009 by Current Medicine Group LLC

The establishment of collaborative sentinel surveillance networks specifically to monitor disease trends among travelers offers new options for evaluating travel health issues. These networks can inform preand posttravel patient management by providing complementary surveillance information, facilitating communication and collaboration between participating network sites, and enabling new analytical options for travel-related research. The two major multinational sentinel networks are TropNetEurop and GeoSentinel. Practical examples demonstrating their significant contributions to improved travel health information are discussed. Data obtained from studying health problems among travelers may also have significant benefits for local populations in resource-limited countries. However, there are limitations of data collected by sentinel networks, so they should be considered as complementary tools and not relied on as an exclusive basis for evaluating health risks among travelers.

\section{Introduction}

In late 2000, travel-related sentinel networks reported two cases of African trypanosomiasis in travelers returning from Tanzania [1,2]. Discussion of the index cases within the networks triggered awareness and led to the rapid recognition of nine similar cases among tourists to the Tarangire and Serengeti National Parks. The temporal clustering of imported cases suggested a change in the local epidemiology of the disease, so local health authorities were advised and instigated control measures to halt the outbreak.

This example highlights the potential benefits of surveillance networks in informing health issues among travelers, and demonstrates how knowledge derived from studying travelers can have important health benefits for local populations in resource-limited countries.

\section{Sentinel Networks}

The establishment of collaborative sentinel surveillance networks in the past 10 years specifically to monitor disease trends among travelers has provided new options for evaluating travel health issues. These networks can augment knowledge through three main mechanisms: 1) they enhance surveillance information by complementing other surveillance activities; 2) they enable rapid communication and dissemination of up-to-date information among providers of clinical care to travelers; and 3) they collect data that provide new methodologic options for travel-related research.

\section{Enhanced Surveillance}

Role of sentinel surveillance and sentinel networks Sentinel surveillance is the collection and analysis of data by designated institutions selected for their geographic location, medical specialty, and ability to accurately diagnose and report high-quality data. Sentinel networks link groups of participating organizations to a central data receiving and processing center. Travel-related sentinel networks specifically target centers with an interest in health problems among immigrants and travelers. They prospectively and systematically collect data to track travel-related diseases.

Sites are recruited to contribute surveillance data on the basis of demonstrated training, experience in recognizing travel-related health events, and reputation in travel and tropical medicine. Regular examination of pooled network data to screen for unexpected increases in notification and to determine the relative frequencies of specific health problems among presenting travelers can also indicate long-term 
geographic and temporal trends, and enables detection of "blips" that might suggest unusual disease activity. Other sentinel surveillance systems exist to monitor disease trends for specific diseases (eg, DengueNet and FluNet established by the World Health Organization), but they are not specific to travelers.

The first major provider-based travel-related surveillance network established was GeoSentinel, which began in 1995 (http://www.istm.org/geosentinel/main.html). Additional and more localized surveillance systems include the European Network on Imported Infectious Disease Surveillance (TropNetEurop), founded in 1999 (http://www.tropnet.net); the German surveillance network for imported infectious diseases (Surveillance Importierter Infektionen in Deutschland, or SIMPID; http://www.simpid.de/); and the National Travel Health Network and Centre ( $\mathrm{NaTHNaC})$, established in 2003 to conduct surveillance on travel-associated illnesses in England, Wales, and Northern Ireland (http://www.nathnac.org) [3].

GeoSentinel and TropNetEurop are the largest of these networks. GeoSentinel is a global network currently comprising 41 geographically dispersed clinics on six continents. All travel-related illnesses are captured by GeoSentinel surveillance via a standardized, computerized system. Ongoing trends are tracked on a month-to-month basis for 60 key diagnoses, and syndromic surveillance is also performed. Enhanced surveillance and notification can be established in outbreaks.

TropNetEurop is an electronic network of more than 50 clinical sites that collect data on infectious diseases imported into Europe. Patient and laboratory data are collected on three main diseases-malaria, dengue fever, and schistosomiasis-but TropNetEurop's members are also encouraged to report anything they consider unusual. Although TropNetEurop's geographic and diagnostic coverage is not as broad as GeoSentinel, this network has the advantage of including most of the major European referral centers for travel medicine.

\section{Travelers as a sentinel population}

A "sentinel" is defined as "one who gives a warning or indicates danger" [4]. Historically, new diseases have often first emerged in resource-limited countries, but initial detection and reporting have been from Western countries because diagnostic capabilities and networks for disseminating information are often not available in developing countries. Examples of such diseases include HIV and Marburg hemorrhagic fever [5•]. Because travelers potentially expose themselves to all types of infectious diseases, studying illnesses among travelers can provide new insights into the presence, distribution, and transmission patterns of infections among local populations in the places they have visited [6-9]. Data collected by sentinel networks may enable identification of rare diseases among travelers, and temporal clustering of imported infections may serve as an early indicator of increased disease activity, possibly suggesting changes in the local epidemiology of infections [9]. Thus, increased knowledge of health problems that occur among sentinel travelers can provide significant insights into circulating health risks among local populations. The surveillance infrastructure and diagnostic technology accessible in developed countries therefore can be the source for identifying new outbreaks or emerging foci of infections for areas with limited public health resources, and is complementary to local surveillance systems. Disease surveillance among travelers may also be the mechanism for detecting new disease syndromes, or may herald emerging resistance patterns among microorganisms.

Examples of travelers as sentinels for emerging new infections predate the establishment of sentinel surveillance networks. The first outbreak of Lassa fever was noted among American missionaries in Nigeria in 1969, and recognition of Lake Malawi as a source of acquisition of schistosomiasis was based on two cases of neuro schistosomiasis among Peace Corps volunteers in 1992 [6].

One of the best examples of the role travelers can play as sentinels for infection is the severe acute respiratory syndrome (SARS) outbreak. SARS was caused by a novel and newly identified coronavirus, which originated in November 2002 in the Guangdong province of China. The infection was transmitted by a physician from mainland China who travelled to Hong Kong and stayed in a local hotel, where he infected guests from other countries. These individuals subsequently spread the virus as they returned to their respective countries, thereby initiating the global SARS epidemic which, during a 7-month period, ultimately involved 27 countries and more than 8000 probable cases [10-15].

The emergence of chikungunya virus provides another example of travelers tracking a disease outbreak. Since 2005 , millions of cases of chikungunya have occurred in countries around the Indian Ocean and in India, and more than 1000 travelers returning to Europe and the United States have been affected. Sequence analysis performed in France on four infected travelers identified that a new virus variant had emerged [16]. Of note, the outbreak was captured by travelers to GeoSentinel sites in France, Switzerland, and Germany.

Results of a recent study on measles exportation from Japan to the United States demonstrated that the trend of exported measles corresponded with concurrent measles activity in Japan, thereby supporting the value of assessing disease trends among travelers to inform local disease trends [17].

\section{Sentinel surveillance systems compared with national notifiable disease surveillance}

From the preceding examples, it is evident that sentinel networks are not a prerequisite for using travelers as sentinels. However, sentinel networks can complement other 
traditional public health surveillance systems, including laboratory surveillance systems and notifiable disease surveillance, to provide supplementary information regarding disease trends in local and traveling populations.

The list of notifiable diseases is often limited, and sentinel surveillance networks can provide a mechanism for capturing information on additional diseases. For example, GeoSentinel requests sites to report on all travel-related illness, and thus captures a very broad scope of diseases. There is no uniformity between countries regarding surveillance infrastructure, health care-seeking behavior, specific diseases requiring notification, case definitions and laboratory methods used, or details of epidemiologic and clinical information captured, so comparison of disease incidence estimates between countries using notification data is problematic $[5 \bullet, 18,19]$. In contrast, surveillance network sites can achieve standardized reporting and have one centralized database, thereby facilitating multinational comparisons. Finally, the problem of underreporting in conventional surveillance systems is well recognized, but because clinics volunteer to contribute to sentinel surveillance efforts, greater compliance with reporting conditions of interest and greater expertise with recognition and diagnosis of travel-related health problems are expected.

However, problems also exist with data collected by sentinel surveillance. Foremost is that only cases presenting to specific clinics involved in data collection are captured, which is only a small proportion of all imported cases of the disease. This is in contrast to national notification systems, which aim to include all notifiable cases. Additionally, centralized laboratory testing for all patients is impossible due to geographic diversity; absolute consistency in diagnostic criteria and recording across all sites cannot be guaranteed.

Cases of imported dengue fever reported to TropNetEurop and GeoSentinel provide an example of the role sentinel networks play in enhancing local disease surveillance. Both sentinel networks have examined data on dengue infection among travelers and have been able to detect trends indicative of changes in disease activity among endemic populations. Although local surveillance mechanisms would also be likely to detect such trends, sentinel network data have been able to preempt local outbreaks $[9,20 \bullet \bullet]$, such as occurred in April 2002 when TropNetEurop and GeoSentinel alerted the international community to a dramatic increase in travel-related dengue from Thailand before official local surveillance data reports emerged [21]. In a study examining seasonality and annual trends of dengue fever among 522 travelers reported to GeoSentinel, the major epidemic peak of travel-related dengue preceded reports of increased dengue activity among local populations, and dengue cases among sentinel travelers at atypical times of the year predicted epidemic activity among inhabitants of endemic areas $[20 \bullet \bullet]$.

\section{Sentinel Networks Facilitate Communication} and Information Dissemination

Rapid flow of reporting information

A major advantage of sentinel surveillance networks is that they primarily rely on a strategy of provider-based reporting, meaning that the reporting physicians have actually seen the patient and can use their expertise to report diagnoses based on clinical suspicion. This reporting mechanism differs from many conventional surveillance programs' reliance on public health laboratories and local health departments for case reporting, often after a substantial time lag [7,22]. Although laboratory testing to confirm diagnoses will usually be performed, clinically based reporting facilitates much more rapid information flow and a faster response time.

\section{Mechanism for rapidly disseminating information about unusual events}

Sometimes individual clinical units are able to detect a single imported case of an unusual disease that is significant, or can recognize an infection that was acquired from a geographically unexpected area. However, detection of an unusual increase in cases of a disease imported from specific countries generally requires several patients, which for tropical diseases may be feasible only by combining surveillance data from several sentinel units [9]. Sentinel networks enable rapid inquiry regarding additional cases among affiliated sites, so if one member site sees an unusual case, they can immediately notify the rest of the network and quickly determine if a pattern can be established. If a suspicious cluster is revealed, an outbreak may or may not be occurring, but it signals that additional examination of disease activity may be warranted. Facilitation of a collegial approach to information sharing between clinical sites is a fundamental aspect of these networks. Additionally, the establishment of communication links between network members provides a structured, efficient mechanism for early dissemination of alerts regarding suspected changes in disease activity. Recognized clusters of events may then be posted onto ProMed (www.promedmail.org) or similar alert systems for wider dissemination of information.

\section{Using Sentinel Network Data for Research Benefits}

Like most surveillance systems, sentinel networks collect relatively limited data fields because they were established to monitor illness in travelers and were not specifically designed as a research tool. However, information is prospectively collected in a standardized way on a large sample of ill travelers who are often diverse in origin, places visited, and types of travel undertaken. Thus, using sentinel networks is beneficial, not just for surveillance 
purposes, but also as a resource for research, outputs of which can be informative for clinicians working in preand posttravel health.

\section{Scope of data}

Many previous studies in travel medicine have been retrospective case reports or case series performed by single institutions using data collected opportunistically rather than systematically. They often involved travelers from a single region, travelers to a single destination, or individuals who developed a single health outcome [23•]. Furthermore, most single centers see too few cases of any particular condition to be able to confidently evaluate disease trends among travelers. The volume of information collected by multicenter surveillance is a major advantage compared with single-site analyses and allows greater statistical power. Examination of GeoSentinel data, for example, enables a multinational description of the spectrum of disease acquired by a broad range of travelers returning from diverse global destinations. Other networks such as TropNetEurop and $\mathrm{NaTHNaC}$ enable inclusion of more comprehensive regional data.

\section{Refined risk-factor identification}

Well-recognized themes and principles are known to underpin travel-associated risks, such as destination, duration, season, activities, and travel conditions that help define risks in the prospective traveler. However, better understanding of the incremental impact of these factors on illness acquisition enables evidenced-based prioritization of risk-minimization strategies according to the individual's specific travel details and provides enhanced guidance for appropriate empiric diagnostic and treatment measures if the traveler becomes ill. Analysis of network surveillance data facilitates determination of odds ratios that indicate associations between illness and demographic, host, itinerary, and travel characteristics.

Estimating risks using proxy denominator data

Although sentinel network data capture only a proportion of cases of disease, significant increases in reported cases in the absence of significant changes to itinerary patterns may indicate that absolute disease risks are changing. Sentinel surveillance networks do not provide denominator data for risk calculations (as discussed further later), but proxy denominator data may be obtainable from other sources. The World Tourism Organization [24] publishes annual numbers of inbound international tourists by geographic region, and some countries also have their own mechanisms for monitoring tourist movements, which can be used to infer denominators. Relative risks of illness according to destinations visited among reported cases can then be determined.

\section{Proportionate morbidity calculations}

There are limitations in using raw numbers of cases reported to sentinel networks to determine risk estimates or trends in disease incidence, but longitudinal data collected by sentinel surveillance systems are useful for documenting trends in proportionate morbidity of infections over time [25•]. Patterns of both syndromic and specific etiologic diagnoses can be examined. Proportionate morbidity calculations require assessment of the number of patients reported with a given diagnosis, and division by the total number of ill travelers seen from each destination in the same time period. This enables standardized calculations of the proportion per 1000 ill returned travelers from each region with diagnoses of interest.

\section{Limitations}

Despite the benefits of using sentinel surveillance data for research, there are also several limitations, especially in terms of inferring absolute risks of disease among travelers. These limitations must be recognized to appreciate the appropriate context in which the data can be used and the likely biases of analytical results.

\section{Sampling frame}

One of the main limitations of network surveillance is that the data represent a convenience sample of sentinel travel sites, and information is collected only on ill travelers who seek medical care from a participating site. In other words, only patients who have become unwell during or after travel, necessitating presentation to a hospital or clinic with a presumed travel-related health problem, are captured. It is estimated that about $8 \%$ of travelers have symptoms warranting presentation to a doctor either during or after return from their travels, but obviously many will present to clinics outside surveillance networks $[26,27]$. Sentinel clinics are likely to see a different patient population than general clinics. Recently returned travelers with unusual, severe, or persistent symptoms, or those who have had prolonged travel, may be more likely to go to sentinel clinics, whereas unwell travelers with common, nonspecific symptoms (eg, fever, respiratory symptoms, or diarrhea) may be more likely to visit a general practitioner. Therefore, patients who have certain demographic characteristics, have travelled to certain exotic destinations, or have symptoms suggestive of an exotic imported illness may be more likely to be referred to a specialized clinic, and this introduces selection and reporting biases [25•].

Additionally, the data collected will be influenced by the geographic distribution and proportionate contributions of individual sites. Travelers who have remained well and required no medical follow-up, or those who have been seen by family doctors, will not be represented. Patients who die while traveling are likely to be excluded. Consequently, the data are not representative of or generalizable to the entire traveling population. 
Inability to calculate incidence rates

Because only a proportion of actual cases are included in sentinel surveillance data, and because sentinel surveillance does not provide external denominator data of the total number of travelers to different regions, it is not possible to use surveillance data to calculate incidence rates or absolute disease risks. Defining attack rates for various infections associated with particular traveler demographics or itineraries would be the ideal data required by travel health practitioners to inform priorities for pre- and posttravel evaluation. This type of information can be assessed accurately only via large cohort studies.

\section{Limitations in trend assessment}

Calculating trends in reported cases of illness must account for changes in travel itineraries, because fluctuations in the frequency of traveler visits to certain destinations over time can influence the expected proportional rates of detection of different illnesses. Without denominator data regarding the total number of travelers to each region, increased numbers of reported cases of illness from a specific geographic area may be due to increased travel to the area or changes in notification behavior rather than reflecting a true increase in transmission risks [9].

\section{Practical Examples of Sentinel Networks} Informing Pretravel and Posttravel Evaluations The role of sentinel networks in providing enhanced surveillance information, improved mechanisms for communication, and as a basis for research promotes understanding of the ways in which these networks enhance pre- and posttravel evaluations. Many of the examples given here also highlight the benefits of providing information that enables timely instigation of preventive health interventions in developing countries.

\section{Impact of identifying case clusters on pretravel recommendations}

Communication between sentinel surveillance sites provides an effective system for rapidly translating new risk information into clinical practice modifications [9]. A cluster of cases in people returning from an area may suggest that heightened disease activity exists, implying that emphasizing and prioritizing precautions during pretravel counseling may be prudent. There are several specific examples of modifying pretravel advice based on sensitive detection of sentinel events in travelers using network information and rapid communication.

In June 1999, TropNetEurop identified a case of Plasmodium falciparum malaria acquired from an area in the Dominican Republic that was thought to be malaria-free and for which malaria chemoprophylaxis was not advised. Discussion of the index case among site members led to recognition of 13 cases among European tourists to the area [28]. Based on these cases, travel recommendations were temporarily amended to include antimalarials and strict antimosquito measures. In November 2004, reports from surveillance networks again emerged of 17 cases of malaria in US, Canadian, and European travelers returning from areas in the Dominican Republic previously considered nonmalarious [29]. Recommendations for chloroquine prophylaxis and timely public health interventions to control the outbreak were again instigated.

GeoSentinel and TropNetEurop were also instrumental in recognizing and disseminating early reports of an outbreak of histoplasmosis that occurred among college students who had traveled to Mexico for spring break in March 2001 [30].

\section{Impact of identifying case clusters on management of returned travelers}

Several examples also demonstrate the value of timely reporting of disease outbreaks by sentinel surveillance networks facilitating immediate dissemination of advice regarding appropriate posttravel evaluation.

GeoSentinel was instrumental in providing early warning of an acute febrile illness among participants in the EcoChallenge Sabah Expedition Race held in Malaysian Borneo in August-September 2000 [31]. Within 24 hours, three GeoSentinel sites in London, New York, and Toronto independently reported clusters of cases with a clinical syndrome suspicious for leptospirosis in nine participants. GeoSentinel was also forwarded information regarding at least 15 other participants with similar illnesses from four other countries (of a total of approximately 300 participants). Advice regarding empiric management of ill, febrile EcoChallenge participants with doxycycline was rapidly circulated. Leptospirosis was later confirmed, with the presumed source being related to severe flooding.

A point source schistosomiasis outbreak affecting 23 Israelis and a German traveler returning from Tanzania was detected in September 2007 and reported by GeoSentinel [32]. It was quickly recognized that the source of the cases was a safari camp near Lake Eyasi. An advisory was immediately issued to clinicians to consider and screen for schistosomiasis in all travelers with potential relevant exposure. The safari camp was also contacted and all bathing activity in the artificial pool thought to be the source of infection was ceased.

\section{Impact of research outputs informing pre- and posttravel evaluations}

Both TropNetEurop and GeoSentinel have published many research papers describing data analyses with potential impacts on clinical management. Use of large databases enables analyses of subgroups of travelers and comparisons across a broad range of destinations. 
TropNetEurop data have provided novel descriptive information on cumulative numbers of cases of dengue fever [33], schistosomiasis [34], falciparum malaria [35], and vivax malaria [36] infections in European travelers and immigrants. For example, these reports have described epidemiologic, demographic, and clinical data on more than 600 cases of Plasmodium vivax, more than 1650 patients with $P$. falciparum, almost 300 patients with imported dengue fever, and more than 300 cases of schistosomiasis. These studies enabled analysis of demographic, itinerary, and clinical risk characteristics associated with disease acquisition. These data can be translated into useful information for clinicians by suggesting comparatively high or comparatively low relative risks of acquiring certain illnesses following travel to particular regions. For example, recent reports of declining numbers of reported cases of $P$. falciparum malaria from the Indian subcontinent perhaps indicate that nonselective prescribing of chemoprophylaxis for visitors to India, Pakistan, Bangladesh, and Sri Lanka should be reconsidered [37].

Several GeoSentinel network analyses have highlighted the utility of identifying risk characteristics in influencing patient management. A paper addressing respiratory infections among GeoSentinel patients showed that the relative frequency of influenza was greatest in travelers visiting friends or relatives and in those whose trip lasted more than 30 days, indicating that these groups should be specifically targeted for pretravel influenza vaccination [38]. It also suggested that increasing age gives travelers a greater comparative risk of pneumonia, suggesting that there should be a lower threshold for early clinical evaluation among older travelers with respiratory symptoms.

Another GeoSentinel study specifically addressed illnesses among people visiting friends and relatives (VFRs) and suggested that immigrant-VFRs were proportionately more likely to have serious or potentially preventable travel-related illnesses such as malaria, typhoid fever, respiratory syndromes, and sexually transmitted infections than tourist travelers [39]. A study examining fever in 6957 unwell travelers returning to GeoSentinel sites found that causes of fever varied by region visited and by time of presentation after travel [40]. Although malaria was the most common specific etiologic diagnosis overall, among travelers to Southeast Asia, dengue was most common. GeoSentinel data have also been used to determine factors associated with acquisition of filarial infection [41] and travel characteristics associated with animal-associated injuries [42]. These types of analyses can assist the clinician in refining appropriate pretravel advice and in guiding posttravel empiric therapy.

Proportionate morbidity calculations can also be used to guide patient management according to destination of travel. The definitive paper describing this methodology involved data on 17,353 ill returned travelers presenting to GeoSentinel sites from different global regions with a variety of diagnoses, and the relative frequencies of different illnesses were compared [25•]. For example, systemic febrile illness without localized findings occurred disproportionately among travelers presenting to GeoSentinel sites following return from sub-Saharan Africa or Southeast Asia. In contrast, acute diarrhea was proportionately more common among those returning from south central Asia, and dermatologic problems were seen particularly among those returning from the Caribbean or Central or South America.

\section{Recognition of emerging trends in microbial resistance patterns}

In addition to clinical reporting of information, dissemination of laboratory data among sentinel network sites may reveal changing patterns in drug resistance among clinical isolates. Because infected travelers carry a large variety of microbial strains from different geographic regions, results of molecular resistance testing of imported isolates can serve as an early warning system for increases in drug resistance. This may have significant practical implications for management of patients.

For example, malaria resistance patterns in endemic areas have been recognized by assessing malaria isolates in ill returned travelers [43]. TropNetEurop has been involved in molecular surveillance of resistance patterns among imported malaria species, with studies assessing the prevalence of resistance to antifolates and chloroquine [44] and to atovaquone/proguanil [45] among P. falciparum isolates imported into Europe.

\section{Conclusions}

Before the establishment of devoted research specific to the traveling population, it was necessary to infer health risks for travelers from local disease information among endemic populations. However, exposures, behaviors, and immunity status among travelers and local inhabitants may be quite different, and the local infrastructure for surveillance of disease trends and emerging disease risks may be far from ideal. Establishing sentinel surveillance networks has several fundamental benefits, including provision of additional complementary surveillance information that may warn of potential health problems among local populations in resource-limited countries. These networks facilitate communication and collaboration between participating network sites. They use travelers as a sentinel population and have demonstrated their potential role in detecting outbreaks and recognizing changing antimicrobial resistance patterns.

Examination of sentinel network data can also provide analytical tools for assessing health problems among travelers, including characterization of risk factors for acquisition of particular diseases and assessment of relative morbidities. The collaborative analysis of multinational 
data collected by sentinel networks such as TropNetEurop and GeoSentinel enhances epidemiologic data quality and facilitates improved evidence-based policies regarding appropriate pre- and posttravel management. Nevertheless, an understanding of the limitations and inherent biases of data collected by sentinel networks is crucial. Thus, although network surveillance is a useful additive tool, it is optimal to combine network surveillance data with other observational and research data for ongoing evaluation of health risks among travelers.

\section{Disclosure}

No potential conflict of interest relevant to this article was reported.

\section{References and Recommended Reading}

Papers of particular interest, published recently, have been highlighted as:

- Of importance

$\bullet \quad$ Of major importance

1. Jelinek T, Bisoffi Z, Bonazzi L, et al.: Cluster of African trypanosomiasis in travelers to Tanzanian national parks. Emerg Infect Dis 2002, 8:634-635.

2. International Society for Infectious Diseases: ProMED-mail Web site. Trypanosomiasis, African-Tanzania. Updated November 6, 2000:20001106.1935. Available at http:// www.promedmail.org. Accessed July 8, 2008.

3. Lawrence J, Jones J, Hill DR: Improving the evidence base for pre-travel advice: the importance of surveillance of travelassociated infection. Br J Gen Pract 2005, 55:566-568.

4. Dorland's Medical Dictionary for Health Consumers. Available at http://medical-dictionary.thefreedictionary. com/sentinel. Accessed July 8, 2008.

5.• Guerin PJ, Grais RF, Rottingen JA, Valleron AJ: Using European travellers as an early alert to detect emerging pathogens in countries with limited laboratory resources. BMC Public Health 2007, 7:8.

This article uses a case study of Shigella dysenteriae serotype 1 (Sd1) in European travelers to highlight the potential role travelers can play as sentinels of infection. It demonstrates how surveillance among travelers from industrialized countries can contribute to earlier detection of emerging diseases in developing countries.

6. Cetron M, Keystone J, Shlim D, Steffen R: Travelers' health. Emerg Infect Dis 1998, 4:405-407.

7. Freedman DO, Woodall J: Emerging infectious diseases and risk to the traveler. Med Clin North Am 1999, 83:865-883.

8. Wilson ME: The traveller and emerging infections: sentinel, courier, transmitter. J Appl Microbiol 2003, 94(Suppl):1S-11S.

9.• Jelinek T, Muhlberger N: Surveillance of imported diseases as a window to travel health risks. Infect Dis Clin North Am 2005, 19:1-13.

Discusses the contribution of TropNetEurop to improving knowledge regarding travelers' health, and provides practical examples. Also discusses some of the benefits and limitations of national and international travel-related sentinel networks.

10. Christian MD, Poutanen SM, Loutfy MR, et al.: Severe acute respiratory syndrome. Clin Infect Dis 2004, 38:1420-1427.

11. Demmler GJ, Ligon BL: Severe acute respiratory syndrome (SARS): a review of the history, epidemiology, prevention, and concerns for the future. Semin Pediatr Infect Dis 2003, $14: 240-244$
12. Galvani AP: Emerging infections: what have we learned from SARS? Emerg Infect Dis 2004, 10:1351-1352.

13. Parashar UD, Anderson LJ: Severe acute respiratory syndrome: review and lessons of the 2003 outbreak. Int J Epidemiol 2004, 33:628-634.

14. Peiris JS, Yuen KY, Osterhaus AD, Stohr K: The severe acute respiratory syndrome. N Engl J Med 2003, 349:2431-2441.

15. Poutanen SM, Low DE: Severe acute respiratory syndrome: an update. Curr Opin Infect Dis 2004, 17:287-294.

16. Parola P, de Lamballerie X, Jourdan J, et al.: Novel chikungunya virus variant in travelers returning from Indian Ocean islands. Emerg Infect Dis 2006, 12:1493-1499.

17. Takahashi H, Saito H: Measles exportation from Japan to the United States, 1994 to 2006. J Travel Med 2008, 15:82-86.

18. Muentener P, Schlagenhauf P, Steffen R: Imported malaria (1985-95): trends and perspectives. Bull World Health Organ 1999, 77:560-566.

19. Ekdahl K, Giesecke J: Travellers returning to Sweden as sentinels for comparative disease incidence in other European countries, campylobacter and giardia infection as examples. Euro Surveill 2004, 9:6-9.

20.• Schwartz E, Weld LH, Wilder-Smith A, et al.: Seasonality, annual trends, and characteristics of dengue among ill returned travelers, 1997-2006. Emerg Infect Dis 2008, 14:1081-1088.

Gives an excellent practical demonstration of the benefits of travelrelated sentinel networks in predicting epidemic dengue activity among inhabitants of endemic areas.

21. International Society for Infectious Diseases: ProMED-mail Web site. Dengue/DHF updates (16). Updated April 26, 2002:20020426.4039. Available at http://www.promedmail. org. Accessed July 8, 2008.

22. Freedman DO, Kozarsky PE, Weld LH, Cetron MS: GeoSentinel: the global emerging infections sentinel network of the International Society of Travel Medicine. J Travel Med 1999, 6:94-98.

23. Leder K, Wilson ME, Freedman DO, Torresi J: A comparative analysis of methodological approaches used for estimating risk in travel medicine. J Trav Med 2008, $15: 263-272$

Discusses the advantages and limitations of using sentinel network data for research, and provides detailed examples of the potential analytical approaches.

24. World Tourism Organization: Tourism Highlights, 2008 Edition. Available at http://www.unwto.org/facts/eng/highlights.htm. Accessed October 22, 2008.

25. Freedman DO, Weld LH, Kozarsky PE, et al.: Spectrum of disease and relation to place of exposure among ill returned travelers. N Engl J Med 2006, 354:119-130.

Demonstrates how sentinel network data can provide proportionate morbidity calculations according to destination of travel that can guide patient management. Also discusses strengths and limitations of using sentinel network data for research purposes.

26. Hill DR: Health problems in a large cohort of Americans traveling to developing countries. J Travel Med 2000, $7: 259-266$.

27. Steffen R, Rickenbach M, Wilhelm U, et al.: Health problems after travel to developing countries. I Infect Dis 1987 , 156:84-91.

28. Jelinek T, Grobusch M, Harms-Zwingenberger G, et al.: Falciparum malaria in European tourists to the Dominican Republic. Emerg Infect Dis 2000, 6:537-538.

29. Centers for Disease Control and Prevention (CDC): Transmission of malaria in resort areas-Dominican Republic, 2004. MMWR Morb Mortal Wkly Rep 2005, 53:1195-1198.

30. International Society for Infectious Diseases: ProMED-mail Web site. Respiratory illness-USA ex Mexico (Acapulco) (04). Updated April 12, 2001:20010412.0727. Available at http://www.promedmail.org. Accessed July 9, 2008. 
31. International Society for Infectious Diseases: ProMED-mail Web site. Leptospirosis-UK, USA, Canada ex Malaysia (Borneo). Updated September 12, 2000:20000912.1553. Available at http://www.promedmail.org. Accessed July 9, 2008.

32. International Society for Infectious Diseases: ProMED-mail Web site. Schistosomiasis-Tanzania (Lake Eyasi). Updated September 4, 2007:20070904.2912. Available at http:// www.promedmail.org. Accessed July 8, 2008.

33. Jelinek T, Muhlberger N, Harms G, et al.: Epidemiology and clinical features of imported dengue fever in Europe: sentinel surveillance data from TropNetEurop. Clin Infect Dis 2002, 35:1047-1052.

34. Grobusch MP, Muhlberger N, Jelinek T, et al.: Imported schistosomiasis in Europe: sentinel surveillance data from TropNetEurop. J Travel Med 2003, 10:164-169.

35. Jelinek T, Schulte C, Behrens R, et al.: Imported Falciparum malaria in Europe: sentinel surveillance data from the European network on surveillance of imported infectious diseases. Clin Infect Dis 2002, 34:572-576.

36. Muhlberger N, Jelinek T, Gascon J, et al.: Epidemiology and clinical features of vivax malaria imported to Europe: sentinel surveillance data from TropNetEurop. Malar J 2004, 3:5.

37. Behrens RH, Bisoffi Z, Bjorkman A, et al.: Malaria prophylaxis policy for travellers from Europe to the Indian Subcontinent. Malar J 2006, 5:7.

38. Leder K, Sundararajan V, Weld L, et al.: Respiratory tract infections in travelers: a review of the GeoSentinel surveillance network. Clin Infect Dis 2003, 36:399-406.
39. Leder $\mathrm{K}$, Tong $\mathrm{S}$, Weld $\mathrm{L}$, et al.: Illness in travelers visiting friends and relatives: a review of the GeoSentinel Surveillance Network. Clin Infect Dis 2006, 43:1185-1193.

40. Wilson ME, Weld LH, Boggild A, et al.: Fever in returned travelers: results from the GeoSentinel Surveillance Network. Clin Infect Dis 2007, 44:1560-1568.

41. Lipner EM, Law MA, Barnett E, et al.: Filariasis in travelers presenting to the GeoSentinel Surveillance Network. PLoS Negl Trop Dis 2007, 1:e88.

42. Gautret P, Schwartz E, Shaw M, et al.: Animal-associated injuries and related diseases among returned travellers: a review of the GeoSentinel Surveillance Network. Vaccine 2007, 25:2656-2663.

43. Schwartz E, Bujanover S, Kain KC: Genetic confirmation of atovaquone-proguanil-resistant Plasmodium falciparum malaria acquired by a nonimmune traveler to East Africa. Clin Infect Dis 2003, 37:450-451.

44. Jelinek T, Peyerl-Hoffmann G, Muhlberger N, et al.: Molecular surveillance of drug resistance through imported isolates of Plasmodium falciparum in Europe. Malar J 2002, 1:11.

45. Wichmann O, Muehlberger N, Jelinek T, et al.: Screening for mutations related to atovaquone/proguanil resistance in treatment failures and other imported isolates of Plasmodium falciparum in Europe. J Infect Dis 2004, 190:1541-1546. 